\title{
SOME PROBLEMS IN CONFORMAL MAPPING
}

\author{
D. C. SPENCER
}

1. Introduction. Attention will be confined to a group of problems centering around so-called schlicht functions - that is, functions regular in a given domain and assuming no value there more than once. The type of problem we consider involves determination of precise bounds for certain quantities depending on the function $f$, as $f$ ranges over the schlicht functions in question. Since, for suitable normalization of the functions at some fixed point of the domain, the resulting family of functions is compact or normal, the extremal schlicht functions always exist and the problem is to characterize them.

Interest was focused on this category of questions by the work of Koebe in the years 1907-1909, who established for the family of functions $f$ of the form $f(z)=z+a_{2} z^{2}+a_{3} z^{3}+\cdots$, schlicht and regular in $|z|<1$, a series of properties, among them the theorem of distortion bearing Koebe's name. This theorem asserts the existence of bounds for the absolute value of the derivative $f^{\prime}(z)$, these bounds depending only on $|z|$. Further efforts were directed toward finding the precise values of the bounds asserted by Koebe's theorem, but success was not attained until 1916 when Bieberbach, Faber, Pick and others gave a final form to the theorem of distortion. At the same time the precise bound for $\left|a_{2}\right|$ was given, namely 2 , and the now famous conjecture was made that $\left|a_{n}\right| \leqq n$ for every $n$. Since 1916 this group of problems has attracted the attention of many, and there is now a considerable literature.

The present state of this sphere of questions will be described briefly in a general sort of way, and a few outstanding problems will be indicated, but no attempt at completeness has been made.

2. The coefficient problem. Let $S$ be the family of functions

$$
f(z)=z+a_{2} z^{2}+a_{3} z^{3}+\cdots
$$

which are regular and schlicht in $|z|<1$. The most famous problem concerning these functions is whether $\left|a_{n}\right| \leqq n(n=2,3, \cdots)$, with equality for any $n$ only in the case when

$$
f(z)=\frac{z}{(1-\eta z)^{2}}=z+2 \eta z^{2}+3 \eta z^{3}+\cdots, \quad|\eta|=1 .
$$

An address delivered before the Los Angeles meeting of the Society on November 30,1946 , by invitation of the Committee to Select Hour Speakers for Far Western Sectional Meetings; received by the editors December 30, 1946. 
It is this problem which has stimulated much of the research leading to the various methods, in particular the method of parametric representation given by Löwner [7 ${ }^{1}$ in 1923 and the recent methods (see $[11,12])$ in which the extremal function is compared with infinitesimal variations more general than those provided by Löwner's method.

There are several short proofs that $\left|a_{n}\right| \leqq n(n=2,3, \cdots)$ when all the coefficients are real, and a simple proof, due to Rogosinski [10], will be sketched here. Without loss of generality we may assume that $f$ is regular and schlicht in $|z| \leqq 1$, for every schlicht function is the limit of such functions. Since $f$ has real coefficients, it takes conjugate values for conjugate values of $z$ and so the map of $|z|<1$ by $f$ is symmetrical with respect to the real axis. Since $f$ is schlicht, $\operatorname{Im} f$ and $\operatorname{Im} z$ have the same sign in $|z| \leqq 1$. The function

$$
\frac{1-z^{2}}{z} f(z)=p(z)
$$

therefore has a positive real part in $|z|<1$ since on the boundary we have, writing $z=e^{i \theta}, \operatorname{Re} p\left(e^{i \theta}\right)=2 \sin \theta \cdot \operatorname{Im} f\left(e^{i \theta}\right) \geqq 0$. It follows that the coefficients of $p$ are majorized by those of the function

$$
\frac{1+z}{1-z}=1+2 z+2 z^{2}+\cdots \text {. }
$$

Since the coefficients of this function as well as the coefficients of the function

$$
\frac{z}{1-z^{2}}=z+z^{3}+z^{5}+\cdots
$$

are positive, we see at once, multiplying both sides of (2.3) by (2.4), that the coefficients of $f$ are majorized by the coefficients of the function

$$
\frac{z}{(1-z)^{2}}=z+2 z^{2}+3 z^{3}+\cdots \text {. }
$$

Similarly, $\left|a_{n}\right| \leqq n$ when $f$ maps $|z|<1$ on a star-like domain. In both cases equality is attained only when $f$ is of the form (2.2). We see here a connection between schlicht functions and functions with positive real part, a connection that will be further emphasized in $\$ 3$ below.

In the general case $\left|a_{n}\right|<e \cdot n$ (see [6(a)]), but the only known pre-

1 Numbers in brackets refer to the bibliography at the end of the paper. 
cise inequalities are: $\left|a_{2}\right| \leqq 2$ and $\left|a_{3}\right| \leqq 3$, with equality in either case only if $f$ has the form (2.2). It is comparatively easy to prove that $\left|a_{2}\right| \leqq 2$, and many proofs of this result are known. The inequality $a_{3} \leqq 3$ lies much deeper, only two essentially different proofs being known. The first proof was given by Löwner [7] in 1923, using his method of parametric representation; the second, recently given, makes use of methods quite different from Löwner's (see [11(a)]).

Although the problem of obtaining precise bounds for the coefficients has attracted the most attention, the central problem of the theory is really the so-called coefficient problem. Given the point $\left(a_{2}, a_{3}, \cdots, a_{n}\right)$, we say that

$$
f(z)=z+b_{2} z^{2}+b_{3} z^{3}+\cdots
$$

belongs to the point $\left(a_{2}, a_{3}, \cdots, a_{n}\right)$ if $f$ is regular and schlicht in $|z|<1$ and if

$$
b_{2}=a_{2}, b_{3}=a_{3}, \cdots, b_{n}=a_{n} .
$$

Conversely we say that the point belongs to $f$. The $n$th region of variability $S_{n}$ is the set of points $\left(a_{2}, a_{3}, \cdots, a_{n}\right)$ in a Euclidean space of $2 n-2$ real dimensions each of which belongs to some $f(z)$. The interior of $S_{n}$ is a bounded, simply-connected domain containing the origin $a_{2}=0, a_{3}=0, \cdots, a_{n}=0$, and $S_{n}$ is the closure of its interior. The only one of these regions which is trivial is $S_{2}$, and it is the circle $\left|a_{2}\right| \leqq 2$.

The coefficient problem is the problem of finding $S_{n}$ for $n=2,3, \cdots$; this problem was introduced by Bieberbach.

A method has recently been developed which characterizes $S_{n}$ for general $n$-explicitly for $n=3$ and implicitly for $n>3 .^{2}$ The boundary of $S_{3}$ may be expressed by equations involving only elementary functions, but for $n>3$ the boundary is complicated and cannot be so expressed. We therefore state the result only for $n=3$.

We begin with the trivial observation that, given any schlicht function $f(z)=z+a_{2} z^{2}+a_{3} z^{3}+\cdots$, the function

$$
e^{-i \theta} f\left(e^{i \theta} z\right)=z+a_{2} e^{i \theta} z^{2}+a_{3} e^{i \theta} z^{3}+\cdots
$$

is also normalized and schlicht. Hence if $S_{3}^{(0)}$ is the subset of $S_{3}$ for which $a_{2}$ is real, the whole of $S_{3}$ is the set of points $\left(a_{2} e^{i \theta}, a_{3} e^{2 i \theta}\right)$, $0 \leqq \theta<\pi$, where $\left(a_{2}, a_{3}\right)$ belongs to $S_{3}^{(0)}$. In other words, $S_{3}$ is obtained from $S_{3}^{(0)}$ by rotations. Since the region $S_{\mathbf{3}}^{(0)}$ is symmetrical with re-

${ }^{2}$ See [11(c)]. The region of variability of $\left(a_{2}, a_{3}\right)$ when $a_{2}$ and $a_{3}$ are both real had been given previously by Peschl [8]. 
spect to the plane $a_{2}=0$, it is sufficient to consider the part of the boundary of $S_{3}^{(0)}$ which lies in the half-space $a_{2} \geqq 0$. This portion of the boundary of $S_{3}^{(0)}$ is made up of the following two analytic surfaces plus their intersection:

(1) Suppose that $0 \leqq \alpha \leqq \pi / 2$. Let $-2(\sin \alpha-\alpha \cos \alpha) \leqq \mu$ $\leqq 2(\sin \alpha-\alpha \cos \alpha)$ and define $\lambda=2 \cos \alpha\{\log (\cos \alpha)-1\}$. Then one of the two surfaces is defined by

$$
\begin{aligned}
& a_{2}=\left(\lambda^{2}+\mu^{2}\right)^{1 / 2} \\
& a_{3}=\lambda^{2}+\mu^{2}+2 \cos \alpha(\lambda-i \mu)+\left(2 \cos ^{2} \alpha+1\right) \frac{\lambda-i \mu}{\lambda+i \mu} .
\end{aligned}
$$

Any function $w=f(z)$ belonging to a point of this surface generally maps $|z|<1$ on the $w$-plane minus a forked slit composed of a ray $\arg (w)=$ constant extending from $w=\infty$ to some finite point where there is a fork composed of two prongs which form angles $2 \pi / 3$ with the ray. On the edge of this surface one or both prongs degenerate to a point. If $\alpha=\pi / 2, w=f(z)$ maps $|z|<1$ on the $w$-plane minus two rays $\arg (w)=$ constant which make an angle $\pi$ at $w=\infty$; in this case $a_{2}, a_{3}$ lie on the parabola $a_{2}=\mu, a_{3}=\mu^{2}-1,0 \leqq \mu \leqq 2$.

(2) Suppose that $0<r<1,-\pi / 2 \leqq \phi \leqq \pi / 2$. Let

$$
\begin{aligned}
\rho^{2} & =1+6 r^{2}+r^{4}+4 r\left(1+r^{2}\right) \cos 2 \phi, \\
\tan \alpha & =\frac{2 r \sin 2 \phi}{1+2 r \cos 2 \phi+r^{2}} \quad\left(-\frac{\pi}{2}<\alpha<\frac{\pi}{2}\right), \\
C_{1} & =\frac{(1+r)^{2}}{2 r} \cos \phi, \quad C_{2}=\frac{(1-r)^{2}}{2 r} \sin \phi, \\
\lambda & =C_{1} \log \frac{\rho}{(1+r)^{2}}+C_{2} \alpha-2 \cos \phi, \\
\mu & =C_{2} \log \frac{\rho}{(1-r)^{2}}-C_{1} \alpha+2 \sin \phi .
\end{aligned}
$$

The second analytic surface is defined by

$$
\begin{aligned}
a_{2}= & \left(\lambda^{2}+\mu^{2}\right)^{1 / 2} \\
a_{3}= & \lambda^{2}+\mu^{2}+\left(C_{1}+i C_{2}\right)(\lambda-i \mu) \\
& +\left(r+\frac{1}{r}+\cos 2 \phi\right) \frac{\lambda-i \mu}{\lambda+i \mu} .
\end{aligned}
$$

If $f(z)$ belongs to a point on this boundary surface of $S_{3}^{(0)}$, then $w=f(z)$ maps $|z|<1$ on the $w$-plane minus a single curved analytic 
slit extending from $w=\infty$ to some finite point. As $r \rightarrow 0$, the slit tends to a straight line arg $(w)=$ constant and the corresponding $f$ tends to $z /\left(1+e^{-i \phi} z\right)^{2}$. As $r \rightarrow 1$,

$$
\lambda \rightarrow 2 \cos \phi\{\log (\cos \phi)-1\}, \quad \mu \rightarrow 2(\sin \phi-\phi \cos \phi) .
$$

That is, $r=1$ corresponds to the edge of intersection of the two surfaces. For functions $w=f(z)$ belonging to this edge, one of the two prongs of the fork is absent.

Even in the case $n=3$ we observe that the equations defining the boundary of $S_{3}$ are sufficiently complicated that it is difficult to infer directly from them that $\left|a_{3}\right| \leqq 3$. Since for $n>3$ the situation is much more complicated, the precise bounds for the coefficients, a specific question about these regions, remain undetermined. Other questions concerning these regions may be asked; for example, what are the maximum and minimum distances of the boundary of $S_{n}$ from the origin (distance from the origin being equal to $\left.\left(\sum_{\nu=2}^{n}\left|a_{\nu}\right|^{2}\right)^{1 / 2}\right)$. In the case of $S_{3}$, the maximum distance of the boundary from the origin is $\left(2^{2}+3^{2}\right)^{1 / 2}=13^{1 / 2}$, attained only for functions of the form (2.2), and the minimum distance of the boundary from the origin is $3^{1 / 2} / 2$, attained only for functions of the form

$$
\frac{z}{1-\eta z / 2^{1 / 2}+\eta^{2} z^{2}}=z+\frac{1}{2^{1 / 2}} \eta z^{2}-\frac{1}{2} \eta^{2} z^{3}+\cdots,|\eta|=1 .
$$

To each boundary point $\left(a_{2}, a_{3}, \cdots, a_{n}\right)$ of $S_{n}$ there belongs one and only one function $w=f(z)$, and this function maps the unit circle on the w-plane minus piecewise analytic slits. Given any complex numbers $p_{2}, p_{3}, \cdots, p_{n}, \sum_{\nu=2}^{n}\left|p_{\nu}\right|^{2}=1$, let

$$
\begin{aligned}
L & =\operatorname{Re}\left(p_{2} a_{2}+p_{3} a_{3}+\cdots+p_{n} a_{n}\right) \\
& =\left(p_{2} a_{2}+p_{2} \bar{a}_{2}+\cdots+p_{n} a_{n}+p_{n} \bar{a}_{n}\right) / 2 .
\end{aligned}
$$

If the maximum value of $L$ in $S_{n}$ is $M$, then $S_{n}$ lies entirely on one side of the $(2 n-3)$-dimensional hyperplane $L=M$. Since $S_{n}$ cannot be convex for $n>2$, such a supporting hyperplane can touch the boundary only at a well-defined subset of boundary points of $S_{n}$. Any function $w=f$ belonging to a point of this subset maps $|z|<1$ on the $w$-plane minus one or more analytic slits meeting at infinity and each of these slits has no finite critical points and is therefore unforked.

If for two different sets of numbers $p_{2}, p_{3}, \cdots, p_{n}$ the corresponding $L$ 's given by (2.9) both have a local extremum at the same boundary point $\left(a_{2}, a_{3}, \cdots, a_{n}\right)$ of $S_{n}$, then the function $f$ belonging to this point is algebraic. These algebraic boundary functions map $|z|<1$ 
onto the plane minus slits. It would be interesting to study the character of algebraic schlicht functions which map onto slit regions.

The method used to characterize $S_{n}$ has been sketched in [11(c)] and a detailed exposition is in course of preparation by A. C. Schaeffer and the author. Therefore, no attempt will be made to describe the method here. We remark only that the starting point of the method is to maximize a certain function $F\left(a_{2}, \bar{a}_{2}, a_{3}, \bar{a}_{3}, \cdots, a_{n}, \bar{a}_{n}\right)$ in $S_{n}$, the maximum being attained at a boundary point $\left(a_{2}, a_{3}, \cdots, a_{n}\right)$. If $f$ belongs to this boundary point, a differential equation for $f$ is obtained by making infinitesimal variations of this extremal function. The method then consists of a study of the resulting differential equation.

In the next section ( $\$ 3)$, Löwner's method of parametric representation will be briefly discussed. This method provides an $\epsilon$-variation of any schlicht function, but the variation is one-sided in that $\epsilon$ can have only one sign. This defect does not occur in the more powerful variational methods recently developed (see [11] and [12]).

3. Relations between coefficients of schlicht functions and coefficients of functions with positive real part. In the case of functions with positive real part, the regions of variability of the coefficients have been obtained by Carathéodory [1], Toeplitz [13], and others. If numbers $\gamma_{1}, \gamma_{2}, \cdots, \gamma_{n-1}$ are given, then there is a function

$$
p(z)=1+2 \sum_{\nu=1}^{\infty} c_{\nu} z^{\nu}
$$

which is regular and has positive real part in $|z|<1$ with

$$
c_{1}=\gamma_{1}, c_{2}=\gamma_{2}, \cdots, c_{n-1}=\gamma_{n-1}
$$

if and only if the Hermitian form

$$
H=\sum_{\mu=1}^{n} \sum_{\nu=1}^{n} \gamma_{\mu-\nu} x_{\mu} \bar{x}_{\nu}
$$

is positive semi-definite, where $\gamma_{0}=1$ and $\gamma_{-k}=\bar{\gamma}_{k}$ is the complex conjugate of $\gamma_{k}$. In geometrical language, there is a function $p(z)$ with $\operatorname{Re} p(z)>0$ in $|z|<1$ if and only if $\left(\gamma_{1}, \gamma_{2}, \cdots, \gamma_{n-1}\right)$ lies inside or on the boundary of the smallest convex region containing the curve $e^{i \theta}, e^{2 i \theta}, \cdots, e^{i(n-1) \theta}(\theta$ real $)$, and the interior of this region is characterized by the property that $H$ is positive definite there. The $n$th region of variability $P_{n}$ is the set of points $\left(c_{1}, c_{2}, \cdots, c_{n-1}\right)$ each of which belongs to some $p(z)$. The points of $P_{n}$ have the one-to-one parametric representation 


$$
\begin{aligned}
c_{k}=\sum_{j=1}^{n-1} \mu_{j} e^{i k \theta_{j}}, & 1 \leqq k \leqq n-1, \mu_{j} \geqq 0, \\
\sum_{j=1}^{n-1} \mu_{j} \leqq 1, & 0 \leqq \theta_{j}<2 \pi,
\end{aligned}
$$

and the boundary of $P_{n}$ is characterized by the condition that $\sum_{j=1}^{n-1} \mu_{j}=1$. To each boundary point defined by (3.3) there belongs only one function, namely

$$
p(z)=\sum_{j=1}^{n-1} \mu_{j} \frac{1+e^{i \theta_{j} z}}{1-e^{i \theta_{j} z}}, \quad \quad \sum_{j=1}^{n-1} \mu_{j}=1 .
$$

Rogosinski [10] has studied the class of functions

$$
f(z)=z+a_{2} z^{2}+a_{3} z^{8}+\cdots
$$

which are regular for $|z|<1$ and have the property that $\operatorname{Im} f$ and Im $z$ have the same sign in $|z|<1$, a property which implies that all the coefficients are real. We have seen that schlicht functions with real coefficients have this property, and the argument used above to prove that $\left|a_{n}\right| \leqq n$ for schlicht functions with real coefficients extends at once to this wider class of power series, a class of functions which Rogosinski has called "typically-real." In fact, if $f$ is typicallyreal then

$$
\frac{1-z^{2}}{z} f(z)=p(z)
$$

is a function of positive real part, and conversely. From this simple relation the variability regions $T_{n}$ for the coefficients of typically-real functions are easily obtained from the corresponding regions $P_{n}$ for functions with positive real part. It is readily seen that $T_{n}$ is the smallest convex region containing the $n$th variability region for schlicht functions all of whose coefficients are real. This raises an interesting question: what is the smallest convex region containing $S_{n}$ itself?

A domain containing $w=0$ is said to be star-like (with respect to $w=0$ ) if any point of it can be joined to the origin by a straight-line segment which lies in the domain. The variability regions $S_{n}^{*}$ for the subfamily of schlicht functions which map $|z|<1$ onto star-like domains are also related to the regions $P_{n}$ in a simple way. This follows from the fact that if $f$ maps $|z|<1$ onto a star-like domain, then

$$
\frac{f(z)}{z f^{\prime}(z)}=p(z),
$$


where $p(z)$ is regular and has positive real part in $|z|<1$. Conversely, if $p(z)$ is regular and has positive real part in $|z|<1$, then

$$
f(z)=z \exp \int_{0}^{z} \frac{1-p(\zeta)}{\zeta p(\zeta)} d \zeta
$$

is regular in $|z|<1$ and maps the unit circle on a star-like domain. Writing

$$
p(z)=1+2 \sum_{p=1}^{\infty} c_{\nu} z^{\nu}
$$

we have

$$
\begin{aligned}
& a_{2}=-2 c_{1}, \quad a_{3}=-c_{2}+4 c_{1}^{2}, \\
& a_{4}=\left(-2 c_{3}+14 c_{1} c_{2}-24 c_{1}^{3}\right) / 3, \cdots .
\end{aligned}
$$

The parametric representation of $P_{n}$ given by (3.3) may be used to define a parametric representation of $S_{n}{ }^{*}$. Let

$$
a_{k}=a_{k}\left(\theta_{1}, \theta_{2}, \cdots, \theta_{n-1} ; \mu_{1}, \mu_{2}, \cdots, \mu_{n-1}\right)
$$

be the $k$ th coefficient of the function

$$
f(z)=\frac{z}{\prod_{\nu=1}^{n-1}\left(1-e^{i \theta_{\nu}}\right)^{2 \mu_{\nu}}}, \quad \mu_{\nu} \geqq 0, \sum_{\nu=1}^{n-1} \mu_{\nu} \leqq 1,
$$

which maps $|z|<1$ on a star-like domain. As the parameters vary over the parameter space, the point $\left(a_{2}, a_{3}, \cdots, a_{n}\right)$ sweeps out $S_{n}{ }^{*}$. The boundary of $S_{n}^{*}$ is characterized by the condition

$$
\sum_{\nu=1}^{n-1} \mu_{\nu}=1
$$

and to each boundary point defined in this way there belongs the unique function given by (3.10). In this case the function (3.10) maps $|z|<1$ on the plane minus $q(1 \leqq q \leqq n-1)$ straight-line slits pointing toward the origin, and adjacent slits form at $\infty$ an angle equal to $2 \mu_{\nu} \pi$.

The intersection of the boundary of $S_{n}$ with $S_{n}{ }^{*}$ is the subset of the boundary of $S_{r}^{*}$ for which

$$
\mu_{\nu}=\frac{m_{\nu}}{k-1}, \quad \sum_{\nu=1}^{n-1} m_{\nu}=k-1,
$$

where $m$, and $k$ are integers, $m_{\nu} \geqq 0,2 \leqq k \leqq n$. 
The method of Löwner [7] provides a deeper connection between schlicht functions and functions with positive real part. Heuristically, the method may be briefly described as follows.

For each $\tau, 0 \leqq \tau \leqq T(T>0)$, let $S(z, \tau), S(0, \tau)=0$, be a schlicht function mapping $|z|<1$ onto a star-like domain $D^{*}$. Then for any $t>0$, the values of the function $e^{-t} S(z, \tau)$ lie inside $D^{*}$ and so the function $\phi=\phi(z, t, \tau)=S^{-1}\left(e^{-t} S(z, \tau), \tau\right)$, where $S^{-1}$ denotes the function inverse to $S$, is regular, schlicht and bounded by 1 in $|z|<1$. We have

$$
\left.\frac{d \phi}{d t}\right|_{t=0}=-\frac{S(z, \tau)}{S^{\prime}(z, \tau)}
$$

and so

$$
S^{-1}\left(e^{-\Delta t} S(z, t), t\right)=z-\frac{S(z, t)}{S^{\prime}(z, t)} \Delta t+o(\Delta t) .
$$

Now let us define a one-parameter family of functions

$$
g(z, t)=\gamma(t)\left(z+a_{2}(t) z^{2}+a_{3}(t) z^{3}+\cdots\right), \quad \gamma(t)>0,
$$

which are regular and schlicht in $|z|<1$ for $0 \leqq t \leqq T$ and such that

$$
g(z, t+\Delta t)=g\left(S^{-1}\left(e^{-\Delta t} S(z, t), t\right), t\right) .
$$

$$
g(z, t+\Delta t)=g(z, t)-z \frac{\partial g(z, t)}{\partial z} p(z, t) \Delta t+o(\Delta t)
$$

where $p(z, t)=S(z, t) /\left(z S^{\prime}(z, t)\right)$ has positive real part in $|z|<1$. Dividing by $\Delta t$ and letting $\Delta t$ approach zero, we obtain the differential equation

$$
\frac{\partial g(z, t)}{\partial t}=-z \frac{\partial g(z, t)}{\partial z} p(z, t) .
$$

If we divide both sides of (3.17) by $z$ and then take $z=0$, we have

$$
\frac{d \gamma(t)}{d t}=-\gamma(t), \quad \gamma(t)=\gamma(0) e^{-t} .
$$

The equation (3.17) may also be given a direct geometrical interpretation. Suppose that $g(z, t)$ and $p(z, t)$ are both regular and $\operatorname{Re} p>0$ in $|z| \leqq 1$ for $0 \leqq t \leqq T$. Taking $z=e^{i \theta}$ in (3.16) we have 


$$
g\left(e^{i \theta}, t+\Delta t\right)=g\left(e^{i \theta}, t\right)+i \frac{\partial g\left(e^{i \theta}, t\right)}{\partial \theta} p\left(e^{i \theta}, t\right) \Delta t+o(\Delta t) .
$$

The map $D_{t}$ of $|z|<1$ by $g(z, t)$ is a domain bounded by an analytic curve; the quantity $i \partial g\left(e^{i \theta}, t\right) / \partial \theta$ has the direction of the inner normal to the boundary of $D_{t}$ at the point $w=g\left(e^{i \theta}, t\right)$ and $i \partial g\left(e^{i \theta}, t\right) / \partial \theta \cdot p\left(e^{i \theta}, t\right)$ is a vector which makes an angle less than $\pi / 2$ with this inner normal. Equation (3.19) states that the boundary point $g\left(e^{i \theta}, t+\Delta t\right)$ of the domain $D_{t+\Delta t}$ lies inside $D_{t}$. Thus, as $t$ increases from 0 to $T$, the domain $D_{t}$ shrinks and, if $t^{\prime}<t^{\prime \prime}, D_{t^{\prime \prime}} \subset D_{t^{\prime}}$. Writing

$$
p(z, t)=1+2 \sum_{\nu=1}^{\infty} c_{\nu}(t) z^{\nu}
$$

and equating coefficients of $z^{n}(n \geqq 2)$ in (3.17), we obtain

$$
\frac{d a_{n}(t)}{d t}+(n-1) a_{n}(t)=-2 \sum_{\nu=1}^{n-1} \nu a_{\nu}(t) c_{n-\nu}(t) .
$$

Integrating this equation between the limits $t^{\prime}$ and $t^{\prime \prime}, 0 \leqq t^{\prime}<t^{\prime \prime} \leqq T$,

$$
e^{(n-1) t^{\prime \prime}} a_{n}\left(t^{\prime \prime}\right)-e^{(n-1) t^{\prime}} a_{n}\left(t^{\prime}\right)
$$

$$
=-2 \int_{t^{\prime}}^{t^{\prime \prime}} e^{(n-1) \tau} \sum_{\nu=1}^{n-1} \nu a_{\nu}(\tau) c_{n-\nu}(\tau) d \tau .
$$

In particular,

$$
\begin{aligned}
& e^{t^{\prime \prime}} a_{2}\left(t^{\prime \prime}\right)-e^{t^{\prime}} a_{2}\left(t^{\prime}\right)=-2 \int_{t^{\prime}}^{t^{\prime \prime}} e^{\tau} c_{1}(\tau) d \tau \\
& \quad a_{3}\left(t^{\prime \prime}\right)-e^{2 t^{\prime}} a_{3}\left(t^{\prime}\right) \\
& \quad=-2 \int_{t^{\prime}}^{t^{\prime \prime}} e^{2 \tau} c_{2}(\tau) d \tau+e^{2 t^{\prime \prime}} a_{2}\left(t^{\prime \prime}\right)^{2}-e^{2 t^{\prime}} a_{2}\left(t^{\prime}\right)^{2} .
\end{aligned}
$$

If the sense of the parameter $t$ is reversed by replacing $t$ by $T-t$, and if (for simplicity) we again write $c_{\nu}(t)$ for $c_{\nu}(T-t), a_{\nu}(t)$ for $a_{v}(T-t)$, the formula (3.22) becomes

$$
\begin{aligned}
& e^{-(n-1) t^{\prime \prime}} a_{n}\left(t^{\prime \prime}\right)-e^{-(n-1) t^{\prime}} a_{n}\left(t^{\prime}\right) \\
& =2 \int_{t^{\prime}}^{t^{\prime \prime}} e^{-(n-1) \tau} \sum_{\nu=1}^{n-1} \nu a_{\nu}(\tau) c_{n-\nu}(\tau) d \tau .
\end{aligned}
$$

Sometimes one, sometimes the other, of these two formulas is more convenient.

The above method establishes a curve $\left(a_{2}(t), a_{3}(t), \cdots, a_{n}(t)\right)$ con- 
necting two points of $S_{n}$, and this curve is the transform of a corresponding curve $\left(c_{1}(\tau), c_{2}(\tau), \cdots, c_{n-1}(\tau)\right)$ in the coefficient region $P_{n}$ of functions having positive real part in $|z|<1$.

In Löwner's formulation (see [7]) the function $p(z, t)$ is restricted to be of the form

$$
p(z, t)=(1+\kappa(t) z) /(1-\kappa(t) z),
$$

where $\kappa(t)$ is a continuous function of $t,|\kappa(t)|=1$. In this case the iteration defined by (3.15) introduces a continually lengthening Jordan-arc slit; that is, if $t^{\prime \prime}>t^{\prime}$, then $D_{t^{\prime \prime}}$ (the map of $|z|<1$ by $\left.g\left(z, t^{\prime \prime}\right)\right)$ is obtained by removal of a slit from $D_{t^{\prime}}$ (the map of $|z|<1$ by $\left.g\left(z, t^{\prime}\right)\right)$. Actually, Löwner's parameter $t$ moves in the opposite sense (corresponding to (3.24)), in which case the inclusion relation between $D_{t^{\prime}}$ and $D_{t^{\prime \prime}}$ is reversed, and he showed that any schlicht function of an everywhere dense set can be connected to the function $f(z)=z$ by a curve corresponding to a function $p(z, t)$ of the form (3.25) which depends continuously on $t$. If the functions $p(z, t)$ are not restricted to be of the form (3.25) and if the continuity in $t$ is dropped, then ${ }^{3}$ it can be shown that any schlicht function can be connected to $f(z)=z$, but it is not known that this can be done using only functions of the form (3.25), even without continuity.

Take $t^{\prime \prime}=T, t^{\prime}=0$ in (3.24), and let the value $T$ of the parameter correspond to $f(z)=z$, in which case $a_{k}(T)=0(k=2,3, \cdots)$. Writing $a_{k}(0)=a_{k}(k=2,3, \cdots)$, we then obtain, by recursion, the formulas :

$$
\begin{aligned}
& a_{2}=-2 \int_{0}^{T} e^{-\tau} c_{1}(\tau) d \tau \\
& a_{3}=-2 \int_{0}^{T} e^{-2 \tau} c_{2}(\tau) d \tau+4\left(\int_{0}^{T} e^{-\tau} c_{1}(\tau) d \tau\right)^{2}, \\
& a_{4}=-2 \int_{0}^{T} e^{-3 \tau} c_{3}(\tau) d \tau+12 \int_{0}^{T} e^{-2 \tau} c_{2}(\tau) d \tau \cdot \int_{0}^{T} e^{-\tau} c_{1}(\tau) d \tau
\end{aligned}
$$

$$
\begin{aligned}
& -4 \int_{0}^{T} \int_{\tau_{1}=\tau}^{T} e^{-2 \tau-\tau_{1}} c_{2}(\tau) c_{1}\left(\tau_{1}\right) d \tau d \tau_{1} \\
& -8\left(\int_{0}^{T} e^{-\tau} c_{1}(\tau) d \tau\right)^{3}
\end{aligned}
$$

${ }^{3}$ An unpublished result of A. C. Schaeffer and the author. 


$$
\begin{gathered}
a_{n}=\sum(-1)^{k} \Gamma_{\alpha_{1} \alpha_{2}} \cdots \alpha_{k} \\
\cdot \int_{\tau_{1}=0}^{T} \int_{\tau_{2}=\tau_{1}}^{T} \cdots \int_{\tau_{k=\tau_{k}-1}}^{T} \exp \left[-\sum_{\nu=1}^{k} \alpha_{\nu} \tau_{\nu}\right] \\
\cdot \prod_{\nu=1}^{k} c_{\alpha_{\nu}}\left(\tau_{\nu}\right) d \tau_{1} d \tau_{2} \cdots d \tau_{k}
\end{gathered}
$$

where

$$
\Gamma_{\alpha_{1} \alpha_{2}} \cdots \alpha_{k}=2^{k}\left(n-\alpha_{1}\right)\left(n-\alpha_{1}-\alpha_{2}\right) \cdots\left(n-\alpha_{1}-\alpha_{2}-\cdots-\alpha_{k}\right),
$$

the $\alpha_{1}, \alpha_{2}, \cdots, \alpha_{k}$ being positive integers with sum $n-1$.

Now the interior points of $S_{n}$ are characterized by the property that bounded functions belong to them. Given an interior point $\left(a_{2}, a_{3}, \cdots, a_{n}\right)$ of $S_{n}$, let the minimum maximum modulus of all functions $f$ belonging to this point be $e^{t}(t \geqq 0)$. The set of points of $S_{n}$ which are representable by the formulas (3.26) to (3.29), in which the $c_{\nu}(\tau)(\nu=1,2, \cdots, n-1)$ are measurable functions of $\tau$, is exactly the set of interior points of $S_{n}$ corresponding to the values $t \leqq T$. If, therefore, we take $T=\infty$ in these formulas, any point of $S_{n}$ may be given this integral representation for suitable choice of the curve $\left(c_{1}(\tau), c_{2}(\tau), \cdots, c_{n-1}(\tau)\right)$. More generally, given any schlicht function $f(z)=z+a_{2} z^{2}+a_{3} z^{3}+\cdots$, there is a function $p(z, \tau)$ $=1+2 \sum_{\nu=1}^{\infty} c_{\nu}(\tau) z^{\nu}$ such that the coefficients $a_{n}(n=2,3, \cdots)$ are given by (3.29). Conversely, given any function $p(z, \tau)$, the coefficients defined by (3.29) belong to a schlicht function. The correspondence between functions $f(z)$ and $p(z, \tau)$ is not one-to-one; in general infinitely many $p(z, \tau)$ correspond to a schlicht function $f$.

To any $f$ which has real coefficients, there is a corresponding $p(z, \tau)$ which has real coefficients. Now if $c_{1}, c_{2}, c_{3}, \cdots$ belong to a function with positive real part, so do the numbers

$$
\operatorname{Re} c_{1}, \operatorname{Re} c_{2}, \operatorname{Re} c_{3}, \cdots
$$

(this follows from the convexity of the family of functions $p$ ). Thus if in (3.29) we replace the $c_{\nu}(\tau)$ by their real parts, the resulting $a_{n}$ belongs to a schlicht function with real coefficients. From this remark, it follows at once that $\left|a_{2}\right| \leqq 2,\left|a_{3}\right| \leqq 3$, for complex coefficients. For example, to show that $\left|a_{3}\right| \leqq 3$, we may suppose without loss of generality that $a_{3}>0$. For, given any schlicht function $f$, we have only to consider the function

$$
e^{-i \theta} f\left(e^{i \theta} z\right)=z+a_{2} e^{i \theta} z^{2}+a_{3} e^{2 i \theta} z^{3}+\cdots
$$

which, for suitable choice of $\theta$, will have a real non-negative third co- 
efficient. But if $a_{3}$ is real we have from (3.27) (with $T=\infty$ )

$$
\begin{aligned}
a_{3}= & -2 \int_{0}^{\infty} e^{-2 \tau} \operatorname{Re} c_{2}(\tau) d \tau+4\left(\int_{0}^{\infty} e^{-\tau} \operatorname{Re} c_{1}(\tau) d \tau\right)^{2} \\
& -4\left(\int_{0}^{\infty} e^{-\tau} \operatorname{Im} c_{1}(\tau) d \tau\right)^{2} \\
\leqq & -2 \int_{0}^{\infty} e^{-2 \tau} \operatorname{Re} c_{2}(\tau) d \tau+4\left(\int_{0}^{\infty} e^{-\tau} \operatorname{Re} c_{1}(\tau) d \tau\right)^{2} .
\end{aligned}
$$

The expression on the right of this inequality is the third coefficient of some function with all coefficients real, and therefore as we have seen bounded by 3 . This argument fails for $a_{4}$.

When the $c_{\nu}(\tau)(\nu=1,2, \cdots)$ are independent of $\tau$, the coefficients given by (3.26)-(3.29) belong to the function

$$
f(z)=e^{T} S^{-1}\left(e^{-T} S(z)\right)
$$

where $S(z) /\left(z S^{\prime}(z)\right)=p(z)=1+\sum_{\nu=1}^{\infty} c_{\nu} z^{\nu}$. In particular, for $T=\infty$, $f(z)=S(z)$ and the formulas reduce to (3.8).

Finally, let $\left(a_{1}(t), a_{3}(t), \cdots, a_{n}(t)\right), 0 \leqq t \leqq T$, be a curve lying on the boundary of $S_{n}$. Then it is obvious that the curve $\left(c_{1}(\tau)\right.$, $\left.c_{2}(\tau), \cdots, c_{n-1}(\tau)\right)$, of which $\left(a_{2}(t), a_{3}(t), \cdots, a_{n}(t)\right)$ is the transform, must lie on the boundary of $P_{n}$, at least for almost all $\tau$. On the other hand, if $\left(a_{2}(0), a_{3}(0), \cdots, a_{n}(0)\right)$ is a boundary point of $S_{n}$ and if $\left(c_{1}(\tau), c_{2}(\tau), \cdots, c_{n-1}(\tau)\right)$ lies on the boundary of $P_{n}$ for every $\tau$, it does not necessarily follow that $\left(a_{2}(t), a_{3}(t), \cdots, a_{n}(t)\right)$ is on the boundary of $S_{n}$ for $0<t \leqq T$. The curve $\left(a_{2}(t), a_{3}(t), \cdots, a_{n}(t)\right)$ lies on the boundary only if $\left(c_{1}(\tau), c_{2}(\tau), \cdots, c_{n-1}(\tau)\right)$ is a curve of special form.

For example, in the case $n=3$, the region $S_{3}$ is bounded by two hypersurfaces $H_{1}$ and $H_{2}$ plus their intersection, where $H_{1}$ and $H_{2}$ are the hypersurfaces generated by rotating the surfaces (2.6) and (2.7) respectively. Let $\left(a_{2}(0), a_{3}(0)\right)$, the initial point of the curve, be an interior point of $\mathrm{H}_{2}$. Then

$$
\begin{aligned}
& e^{t} a_{2}(t)=a_{2}(0)-2 \int_{0}^{t} e^{\tau} e^{i \phi(\tau)} d \tau \\
& e^{2 t} a_{3}(t)=a_{3}(0)-a_{2}(0)^{2}+e^{2 t} a_{2}(t)^{2}-2 \int_{0}^{t} e^{2 \tau} e^{2 i \phi(\tau)} d \tau
\end{aligned}
$$

is a curve lying on $H_{2}$, only if $e^{i \phi(\tau)}$ satisfies the equation 


$$
\operatorname{Im}\left\{A_{0} e^{-\tau} e^{i \phi(\tau)}+B_{0} e^{2 i \phi(\tau)}\right\}=0,
$$

where $A_{0}$ and $B_{0}$ are numbers which can be given explicitly in terms of the point $\left(a_{2}(0), a_{3}(0)\right)$. As $t$ tends to infinity, $\left(a_{2}(t), a_{3}(t)\right)$ tends to $\left(2 \eta, 3 \eta^{2}\right)$ for some $\eta,|\eta|=1$, a point on the intersection of $H_{1}$ and $H_{2}$ belonging to the function $f(z)=z /(1-\eta z)^{2}$. No interior point of $\mathrm{H}_{2}$ can be connected to a point on the intersection of $H_{1}$ and $H_{2}$ by a curve corresponding to a finite range of the parameter $t$.

Next, set

$$
\begin{aligned}
e^{t} a_{2}(t) & =-2-2 \int_{0}^{t} e^{\tau} c_{1}(\tau) d \tau \\
e^{2 t} a_{3}(t) & =-1+e^{2 t} a_{2}(t)^{2}-2 \int_{0}^{t} e^{2 \tau} c_{2}(\tau) d \tau
\end{aligned}
$$

and take

$$
\begin{aligned}
& c_{1}(\tau)=\left\{\begin{array}{lr}
e^{i \alpha(\tau)}, & 0 \leqq \tau \leqq t_{0}, \\
\cos \alpha(\tau)+i C, & t_{0}<\tau<\infty,
\end{array}\right. \\
& c_{2}(\tau)=\left\{\begin{array}{lr}
e^{2 i \alpha(\tau)}, & 0 \leqq \tau \leqq t_{0}, \\
\cos 2 \alpha(\tau)+2 i C \cos \alpha(\tau), & t_{0}<\tau<\infty,
\end{array}\right.
\end{aligned}
$$

where $\cos \alpha(\tau)=e^{-\tau}, 0 \leqq \alpha(\tau)<\pi / 2, C$ is a constant, $0 \leqq C \leqq 1$, and $t_{0}$ is uniquely defined by $\sin \alpha\left(t_{0}\right)-\alpha\left(t_{0}\right) \cos \alpha\left(t_{0}\right)=C$. The function $p(z, \tau)$ corresponding to this curve is

$$
p(z, \tau)= \begin{cases}\frac{1+e^{i \alpha(\tau)} z}{1-e^{i \alpha(\tau)} z}=\frac{1+2 i \sin \alpha(\tau) z-z^{2}}{1-2 \cos \alpha(\tau) z+z^{2}}, & 0 \leqq t \leqq t_{0}, \\ \frac{1+2 i C z-z^{2}}{1-2 \cos \alpha(\tau) z+z^{2}}, & t_{0}<\tau<\infty .\end{cases}
$$

For $t=0,\left(a_{2}(0), a_{3}(0)\right)=(-2,3)$; this point belongs to the function $f(z)=z /(1+z)^{2}$. As $t$ increases from 0 to $t_{0}$, the point $\left(a_{2}(t), a_{3}(t)\right)$ moves on the intersection of $H_{1}$ and $H_{2}$; as $t$ increases from $t_{0}$ to $\infty$, $\left(a_{2}(t), a_{3}(t)\right)$ moves on $H_{1}$ and as $t$ tends to infinity approaches the point $\left(-2 i C, 1-4 C^{2}\right)$ belonging to $f(z)=z /\left(1+2 i C z-z^{2}\right)$. Any point on $H_{1}$, or on the intersection of $H_{1}$ and $H_{2}$, is representable in one of the two forms $\left(a_{2}(t) e^{i \theta}, a_{3}(t) e^{2 i \theta}\right),\left(\bar{a}_{2}(t) e^{-i \theta}, \bar{a}_{3}(t) e^{-2 i \theta}\right)$, for suitable choice of $\theta, C$ and $t, 0 \leqq t \leqq \infty$. That is, any point on $H_{1}$, or any point on the intersection of $H_{1}$ and $H_{2}$, may be obtained from (3.32) by a rotation, 
or by a reflection on the axis-plane $\operatorname{Im} a_{2}=\operatorname{Im} a_{3}=0$, or by both.

We observe that the points $\left(-2 i C, 1-4 C^{2}\right), 0 \leqq C \leqq 1$, which lie on the boundary of $S_{n}{ }^{*}$, correspond to $t=\infty$. Rotating through the angles $\pm \pi / 2$, these points become $\left(2 C, 4 C^{2}-1\right),-1 \leqq C \leqq 1$, and they lie on the parabola $a_{3}=a_{2}^{2}-1,-2 \leqq a_{2} \leqq 2$. This parabola plus its rotations is the intersection of the boundary of $S_{3}$ with $S_{3}{ }^{*}$. The parabola is obtained from (3.32) by taking $c_{1}(\tau)=-1, c_{2}(\tau)=1$. As $t$ tends to infinity, $\left(a_{2}(t), a_{3}(t)\right)$ approaches the point $(2,3)$ belonging to $f(z)$ $=z /(1-z)^{2}$, a rotation of the function $z /(1+z)^{2}$ corresponding to $t=0$. Hence every point of $H_{1}$ can be connected to this function, or to a rotation of it, by a curve corresponding to a finite range of the parameter. This is a consequence of the fact that for functions $w=f$ belong to $H_{1}$, the portion of the boundary in the $w$-plane near $w=\infty$ is a straight line arg $(w)=$ constant (see $\$ 2$ ).

The form of these curves lying on the boundary of $S_{3}$ has been calculated from the formulas (2.6) and (2.7). A derivation based on Löwner's method alone would be interesting.

\section{Maps of the coefficient regions $S_{n}$. Let}

$$
U_{n}=T\left(S_{n}\right)
$$

be a continuous mapping of $S_{n}$ on a region $U_{n}$. When $U_{n}$ has the same dimensionality as $S_{n}$, a common type of map has the special form in which, if $\left(b_{2}, b_{3}, \cdots, b_{n}\right)$ is the image of the point $\left(a_{2}, a_{3}, \cdots, a_{n}\right)$ of $S_{n}$, then

$$
b_{\nu}=b_{\nu}\left(a_{2}, a_{3}, \cdots, a_{\nu}\right) ;
$$

that is, $b_{\nu}$ depends only on the coefficients $a_{2}, a_{3}, \cdots, a_{\nu}$.

This type of map frequently arises when we consider the coefficients of the powers of $z$ of some functional $F$ of $f$,

$$
g=F(f),
$$

where $g$ depends on $z$ in an analytic way. Thus we may take

$$
g=(f(z))^{k}=z^{k-1}\left(z+b_{2} z^{2}+b_{3} z^{3}+\cdots\right) .
$$

In the case $k=-1$,

$$
b_{2}=-a_{2}, b_{3}=a_{2}^{2}-a_{3}, b_{4}=2 a_{2} a_{3}-a_{2}^{8}-a_{4}, \cdots
$$

or, generally, 


$$
\begin{aligned}
b_{\nu+1}=\sum_{k_{1} \geqq 0, k_{2} \geq 0, \cdots, k_{\nu} \geq 0, k_{1}+2 k_{2}+\cdots+\nu k_{\nu}=\nu}(-1)^{k_{1}+k_{2}+\cdots+k_{\nu}} & \frac{\left(k_{1}+k_{2}+\cdots+k_{\nu}\right) !}{k_{1} ! k_{2} ! \cdots k_{\nu} !} a_{2}^{k_{1}} \cdots a_{\nu+1}^{k_{\nu}} .
\end{aligned}
$$

We also obtain a mapping (4.2) if we set

$$
g=\frac{f(z)}{f^{\prime}(z)}=z+b_{2} z^{2}+b_{3} z^{3}+\cdots
$$

which gives

$$
b_{2}=-a_{2}, b_{3}=2\left(a_{2}^{2}-a_{3}\right), b_{4}=7 a_{2} a_{3}-4 a_{2}^{3}-3 a_{4}, \cdots .
$$

Another important case of (4.2) is defined by

$$
f_{k}(z)=\left\{f\left(z^{k}\right)\right\}^{1 / k}=z+b_{2} z^{k+1}+b_{3} z^{2 k+1}+\cdots,
$$

$k$ being a positive integer. Here

$$
\begin{gathered}
b_{2}=\frac{a_{2}}{k}, \quad b_{3}=\frac{1}{k}\left(a_{3}-\frac{k-1}{2 k} a_{2}^{2}\right) \\
b_{4}=\frac{1}{k}\left(a_{4}-\frac{k-1}{k} a_{2} a_{3}+\frac{(k-1)(2 k-1)}{6 k^{2}} a_{2}^{3}\right), \cdots .
\end{gathered}
$$

The function $f_{k}$ is schlicht (conversely if a function $f_{k}$ of the form (4.8) is schlicht, so is the function $\left.\left\{f_{k}\left(z^{1 / k}\right)\right\}^{k}\right)$ and hence (4.9) is a mapping of $S_{n}$ on a subset of itself. Finally let

$$
z=f^{-1}(w)=w+b_{2} w^{2}+b_{3} w^{3}+\cdots
$$

where

$$
\text { (4.11) } b_{2}=-a_{2}, b_{3}=2 a_{2}^{2}-a_{3}, b_{4}=5 a_{2} a_{3}-5 a_{2}^{3}-a_{4}, \cdots \text {. }
$$

The maps defined by (4.5), (4.7), (4.9) and (4.11) have been studied by various authors, mainly from the point of view of obtaining precise upper bounds for the coefficients $b_{\nu}$. In the case (4.5), precise bounds are known only for $\nu=2,3$ and 4 , namely, $\left|b_{2}\right| \leqq 2,\left|b_{3}\right| \leqq 1$, $\left|b_{4}\right| \leqq 2 / 3$ (see $[12(\mathrm{a})]$ and $[3(\mathrm{c})]$ ). In this case the conjecture has been suggested that $\left|b_{n}\right| \leqq 2 /(n-1)$.

In the case $(4.7)$, it is obvious that $\left|b_{\nu}\right| \leqq 2(\nu=2,3, \cdots)$ when $f$ maps $|z|<1$ on a star-like domain. However, there are points $\left(a_{2}, a_{3}, a_{4}\right)$ of $S_{4}$ outside $S_{4}{ }^{*}$ for which $\left|b_{4}\right|>2$ (see [11(b)]) and this in 
fact occurs even for points $\left(a_{2}, a_{3}, a_{4}\right)$ belonging to schlicht functions with all coefficients real. The region $B_{n}$ of points $\left(b_{2}, b_{3}, \cdots, b_{n}\right)$ defined by (4.7) was studied by Peschl [8] in preference to $S_{n}$ itself, for the reason that $B_{n}$ has a slightly simpler relationship to $P_{n}$ than $S_{n}$ has.

In (4.9) precise bounds of $b_{\nu}$ are known only for $\nu=2,3$. It is clear that $\left|b_{2}\right| \leqq 2 / k$, and the precise inequality for $b_{3}$ is (see [2])

$$
\left|b_{3}\right| \leqq \frac{2}{k} \exp \left\{-\frac{2(k-1)}{k+1}\right\}+\frac{1}{k} .
$$

The precise upper bounds for the $b_{\nu}$ defined by (4.11) were obtained by Löwner [7] using his method of parametric representation (these bounds may also be obtained in a different way (see $[11(b)])$ ). The inequalities are:

$$
\left|b_{\nu}\right| \leqq \frac{1 \cdot 3 \cdot 5 \cdots(2 \nu-1)}{1 \cdot 2 \cdot 3 \cdots(\nu+1)} 2^{\nu} \quad(\nu=2,3, \cdots)
$$

Equality is attained for any $\nu$ only if $f(z)=z /(1-\eta z)^{2},|\eta|=1$.

Of particular importance is the case in which the mapping (4.2) is linear in $a_{2}, a_{3}, \cdots, a_{\nu}$, that is when

$$
b_{\nu}=e_{1}+e_{2} a_{2}+\cdots+e_{\nu} a_{\nu}
$$

A linear mapping of this type arises whenever we consider the coefficients of the powers of $\zeta$ in the expansion

$$
g(\zeta)=f(\phi(\zeta))=b_{1} \zeta+b_{2} \zeta^{2}+b_{3} \zeta^{3}+\cdots
$$

where

$$
z=\phi(\zeta)=\beta_{1} \zeta+\beta_{2} \zeta^{2}+\beta_{3} \zeta^{3}+\cdots
$$

is regular in a neighborhood of $\zeta=0$. Writing

$$
\{\phi(\zeta)\}^{k}=\sum_{\nu=k}^{\infty} \beta_{\nu}^{(k)} \zeta^{\nu}
$$

we see that

$$
b_{\nu}=\beta_{\nu}^{(1)}+\beta_{\nu}^{(2)} a_{2}+\beta_{\nu}^{(3)} a_{3}+\cdots+\beta_{\nu}^{(\nu)} a_{\nu} \quad(\nu=1,2, \cdots)
$$

where $\beta_{\nu}^{(1)}=\beta_{\nu}, \beta_{\nu}^{(\nu)}=\beta_{1}^{\nu}$.

Suppose first that $\beta_{1}=1$ in (4.14) and let $z=\phi(\zeta)$ map some domain 
$G$ on $|z|<1 .{ }^{4}$ Then the variability region of $\left(b_{2}, b_{3}, \cdots, b_{n}\right)$ is the $n$th coefficient region $S_{n}(G)$ of functions regular and schlicht in $G$.

Roughly speaking, the coefficient problem is easier the larger $G$ is. ${ }^{5}$ For example, let $\lambda$ be a small number, $0<\lambda<1$, and let $G=G(\lambda)$ be the exterior of the circle orthogonal to the real axis and cutting it at $\lambda(1+\lambda)^{-1}$ and $\lambda(1-\lambda)^{-1}$. Then if $\lambda=\lambda_{n}$ is small enough, the precise upper bound for $b_{n}$ can be determined (see [11(b)]). However, these regions depend on $n$.

In particular, consider the class $C$ of domains $G$ which are generated from the unit circle by the family of normalized schlicht functions $f$. The interior of the unit circle itself belongs to $C$, and we denote it by $E$. If $S(G)$ is the family of functions (4.13) which are regular and schlicht in $G$, we define

$$
\alpha_{n}=\alpha_{n}(G)=\sup _{g \subset S(G)}\left|b_{n}\right|
$$

and write

$$
\gamma_{n}=\inf _{G \subset C} \alpha_{n}(G), \quad \Gamma_{n}=\sup _{G \subset C} \alpha_{n}(G) .
$$

Then (see $[11(\mathrm{a})])$

$$
\alpha_{n}(E)=\gamma_{n} .
$$

But, given $n$, there are other domains $G$ (depending on $n$ ) for which $\alpha_{n}=\gamma_{n}$. Moreover, if $\alpha_{n}(E)=n(n=2,3, \cdots)$, we have $\Gamma_{n}=4^{n-1}$ $(n=2,3, \cdots)$. Hence $\alpha_{n}(E)=n(n=2,3, \cdots)$ implies that the inequality

$$
n \leqq \alpha_{n}(G) \leqq 4^{n-1}
$$

is valid throughout $C$.

Let us drop the restriction that $\beta_{1}=1$, and suppose instead that $\phi(\zeta)$ is regular and bounded by 1 in $|\zeta|<1$. The family of functions $g(\zeta)$ given by (4.13) as $f$ and $\phi$ vary is then the family of functions which are subordinate to schlicht functions, and the region of variability of the point $\left(b_{2}, b_{3}, \cdots, b_{n}\right)$ contains $S_{n}$. Concerning precise bounds for the $b_{v}$, it is known only that $\left|b_{2}\right| \leqq 2$. However, if $f$ (in

4 The domain may contain $\infty$. We suppose that $G$ is schlicht although a more general problem is obtained by allowing $G$ to be a multiply-connected Riemann domain. It is also clear that we may formulate the coefficient problem for points of $G$ other than the origin.

${ }^{5}$ If $G$ is the whole plane minus a single point (in which case no function $\phi(\zeta)$ exists), there is only one normalized function $g(\zeta)$ which is regular and schlicht in $G$; in this case the problem is completely trivial. 
(4.13)) has real coefficients or maps $|z|<1$ on a star-like domain, then $\left|b_{\nu}\right| \leqq \nu(\nu=2,3, \cdots)$ (see [6(b)], where an excellent exposition of this family is given).

5. Variability regions in general. Let $\gamma$ be a closed set in $|z|<1$, and let

$$
F=F\left(f(z), \overline{f(z)}, f^{\prime}(z), \overline{f^{\prime}(z)}, \cdots, f^{(n)}(z), \overline{f^{(n)}}(z) \mid \gamma\right)
$$

be a functional depending on the values taken by $f(z), f^{\prime}(z), \cdots, f^{(n)}(z)$ in $\gamma$. The value of $F$ is a number which may be complex, and we suppose that $F$ is defined and continuous whenever $f$ belongs to the family $S$ of schlicht functions. Moreover, writing $f^{(n)}(z)=x_{\mu}+i y_{\mu}$, let $F$ be "differentiable" (for example, in the sense of Volterra) with respect to $x_{\mu}$ and $y_{\mu}(\mu=0,1, \cdots, n)$ when the functions are regarded as independent and only one of them is given an increment. However, we do not need to be precise since we shall be concerned here only with specific and rather trivial examples of functionals $F$. Now let $U=\left(U_{1}, U_{2}, \cdots, U_{m}\right)$ be the point in euclidean space defined by $m$ functionals of this type:

$$
U_{k}=F_{k}\left(f(z), \overline{f(z)}, \cdots, f^{(n)}(z), \overline{f^{(n)}(z)} \mid \gamma_{k}\right) .
$$

The region of variability $V$ of $U$ is the closed set of points $U$ as $f$ ranges over the family $S$ of schlicht functions.

Thus, the $n$th region $S_{n}$ of the coefficients is obtained by taking $U_{k}=f^{(k)}(0) / k !(k=2,3, \cdots, n)$. On the other hand the region of variability of the point

$$
\left(\frac{f^{\prime \prime}(z)}{f^{\prime}(z)}, \frac{f^{\prime \prime \prime}(z)}{f^{\prime}(z)}, \ldots, \frac{f^{(n)}(z)}{f^{\prime}(z)}\right)
$$

for fixed $z, 0 \leqq|z|<1$, is a linear map of $S_{n}$ of the type (4.12). For let

$$
g(\zeta)=f\left(\frac{\zeta+z}{1+\bar{z} \zeta}\right)=\sum_{\nu=1}^{\infty} a_{\nu}\left(\frac{\zeta+z}{1+\bar{z} \zeta}\right)^{\nu}=\sum_{\nu=0}^{\infty} \alpha_{\nu} \zeta^{\nu},
$$

where $\alpha_{0}=g(0)=f(z), \alpha_{1}=g^{\prime}(0)=f^{\prime}(z)\left(1-|z|^{2}\right)$. Then the function

$$
\frac{g(\zeta)-\alpha_{0}}{\alpha_{1}}=\sum_{\nu=1}^{\infty} \frac{\alpha_{\nu}}{\alpha_{1}} \zeta_{\nu}
$$

is a normalized schlicht function, and so the point $\left(\alpha_{2} / \alpha_{1}, \alpha_{3} / \alpha_{1}, \cdots\right.$, $\alpha_{n} / \alpha_{1}$ ) belongs to $S_{n}$. We have

$$
f(\zeta)=g\left(\frac{\zeta-z}{1-\bar{z} \zeta}\right)=\sum_{\nu=0}^{\infty} \alpha_{\nu}\left(\frac{\zeta-z}{1-\bar{z} \zeta}\right)^{\nu}, \quad f^{(\nu)}(z)=\sum_{k=1}^{\nu} \alpha_{k} K(k, \nu, z)
$$


where $K(k, \nu, z)$ depends only on $k, \nu$ and $z$; hence

$$
\frac{f^{(\nu)}(z)}{f^{\prime}(z)}=\left(1-|z|^{2}\right) \sum_{k=1}^{\nu} \frac{\alpha_{k}}{\alpha_{1}} K(k, \nu, z) \quad(\nu=2,3, \cdots) .
$$

If $U=f(z), z$ fixed, the region of variability is defined by

$$
\left|\log \frac{f(z)}{z}+\log \left(1-|z|^{2}\right)\right| \leqq \log \frac{1+|z|}{1-|z|}
$$

(see $[4,3(d)]$ ), where $\log (f(z) / z)$ is the branch which vanishes for $z=0$. Taking $U=f(z) / f^{\prime}(z)$, the corresponding region $V$ is given by

$$
\left|\log \left(\frac{f(z)}{z f^{\prime}(z)}\right)\right| \leqq \log \frac{1+|z|}{1-|z|}
$$

(see $[5,3(b)]$ ), where $\log \left(f(z) / z f^{\prime}(z)\right)$ is the branch vanishing for $z=0$. From (5.3) and (5.4) we obtain at once the two classical inequalities

$$
\begin{aligned}
& \frac{|z|}{(1+|z|)^{2}} \leqq|f(z)| \leqq \frac{|z|}{(1-|z|)^{2}}, \\
& \frac{1-|z|}{(1+|z|)^{3}} \leqq\left|f^{\prime}(z)\right| \leqq \frac{1+|z|}{(1-|z|)^{3}} .
\end{aligned}
$$

If we choose $U=\arg f^{\prime}(z)\left(\arg f^{\prime}(0)=0\right)$ then

$$
\left|\arg f^{\prime}(z)\right| \leqq\left\{\begin{array}{lr}
4 \arcsin |z|, & |z| \leqq 1 / 2^{1 / 2}, \\
\pi+\log \frac{|z|^{2}}{1-|z|^{2}}, & 1 / 2^{1 / 2}<|z|<1 .
\end{array}\right.
$$

It is worth noting that although (5.6) was the starting point of these investigations, (5.7) was not proved until twenty years later when Golusin [3(a)] established it by using Löwner's method. Inequalities (5.6) and (5.7) constitute what are called the distortion and rotation theorems.

An interesting problem arises if we set $U=\left(|f|,\left|f^{\prime}\right|\right)$. This problem has been solved by Robinson [9].

We may also take $U=(f(z), f(-z))$, or $U=\left(f^{\prime}(z), f^{\prime}(-z)\right)$, where $z$ is a fixed point of $|z|<1$. Aspects of this problem have recently been studied by Golusin (see, for example, [3(e), (f), (g) ]).

Another type of problem is obtained by setting

$$
U=\int_{-\pi}^{\pi}\left|f\left(r e^{i \theta}\right)\right|^{\lambda} d \theta, \quad \lambda>0,0<r<1 .
$$


In this case the precise upper bound is unknown. Or take

$$
U=\int_{0}^{r} \int_{-\pi}^{\pi}\left|f^{\prime}\left(\rho e^{i \theta}\right)\right|^{2} \rho d \rho d \theta=\pi \sum_{n=1}^{\infty} n\left|a_{n}\right|{ }^{2} r^{2 n}
$$

Here $U$ is the area of the map of $|z|<r$ by $f$. It is obvious that this area is not less than $\pi r^{2}$ (with equality only if $f=z$ ), but the precise upper bound is not known.

A rough classification of the above types of problems is provided by the order $n$ of the highest derivative involved. The existing literature (apart from papers on the coefficient problem) is mainly concerned with problems involving first and second derivatives only.

6. Generalizations. Two natural generalizations are obtained by: (i) relaxing the restrictions that the domain $G_{z}$ of the variable $z$ is schlicht and simply-connected; (ii) relaxing the restriction that the domain $G_{w}$ of values $w=f(z)$ is schlicht. Both have been considered. We shall comment briefly on these two generalizations, and we discuss (i) first.

For simplicity, let us suppose that $G_{z}$ is schlicht but possibly multiply-connected. We may then consider extremal properties of the family of functions $f(z)$ which are regular, single-valued and schlicht in $G_{z}$ and which are properly normalized at some interior point $z_{0}$ of the region. Problems of this type have been investigated in numerous papers of H. Grötzsch. ${ }^{6}$ The method used by Grötzsch was simplified and improved by $\mathrm{H}$. Grunsky [5], and was later simplified further by G. Golusin ( $[3(b)]$ ) who applied it to a series of problems in the schlicht conformal mapping of multiply-connected regions. In some instances these investigations have led to new and more complete results for simply-connected regions:

The method used is one of comparison with functions which map $G_{z}$ onto the $w$-plane slit along finite arcs of logorithmic spirals which are obtained from the equation $\operatorname{Im}\left(e^{-i \theta} \log w\right)=c$ by holding $\theta$ fixed and varying $c$. Similar comparisons can be made with functions which map $G_{z}$ on the plane minus parallel straight-line slits.

More recently, problems of this type have been successfully attacked by variational methods in which the comparison functions are $\epsilon$-variations of the extremals (see $[12(b)]$ ).

A generalization of type (ii) is obtained by considering the family of $p$-valent functions $f$ which are regular in $|z|<1$ and normalized in some way at $z=0$ and which take no value in $|z|<1$ more than $p$

- See Berichte der Akademie der Wissenschaften, Leipzig, 1928-1932. 
times, where $p$ is some fixed positive integer. Although these functions have been intensively studied, few precise bounds for the family are known when $p>1$; most results obtained so far are statements about order of magnitude only.

The concept of $p$-valency may be generalized to fractional values of $p$ as follows. Let $w=f(z)$ be regular in $|z|<1$, and let $A(R)$ denote the area (regions covered multiply being counted multiply) of that portion of the map of the unit circle by $w=f(z)$ which lies in the circle $|w| \leqq R$. If

$$
A(R) \leqq p \pi R^{2}
$$

for all $R>0$, where $p$ is a positive (though not necessarily integral) number, we say that $f$ is mean $p$-valent. With suitable normalization at $z=0$, the family of mean $p$-valent functions ( $p$ fixed) is compact, and it can be shown that

$$
\begin{aligned}
|f(z)| & \leqq A(1-|z|)^{-2 p} \\
\left|a_{n}\right| & \leqq B n^{2 p-1}
\end{aligned}
$$$$
(p>0),
$$$$
(p>1 / 4) \text {, }
$$

where $A$ and $B$ are constants. (Here $a_{n}$ is the coefficient of $z^{n}$ in the expansion about $z=0$.)

The most interesting class of mean $p$-valent functions is the class of mean 1-valent functions $f$ of the form

$$
f(z)=z+a_{2} z^{2}+a_{3} z^{3}+\cdots .
$$

For this family $\left|a_{2}\right| \leqq 2$ with equality only if $f=z /(1-\eta z)^{2},|\eta|=1$, but there are mean 1-valent functions $f$ for which $\left|a_{3}\right|>3 .{ }^{7}$ However, these functions resemble schlicht functions so far as orders of magnitude are concerned.

\section{REFERENCES}

1. C. Carathéodory, Über den Variabilitätsbereich der Fourier'schen Konstanten von positiven harmonischen Funktionen, Rend. Circ. Mat. Palermo vol. 32 (1911) pp. 193-217.

2. M. Fekete and G. Szegö, Eine Bemerkung über ungerade schlichte Funktionen, J. London Math. Soc. vol. 8 (1933) pp. 85-89.

3. G. M. Golusin, (a) Sur les théorèmes de rotation dans la thêorie des fonctions univalentes, Rec. Math. (Mat. Sbornik) N.S. vol. 1 (1936) pp. 293-296.

(b) Über die Verzerrung bei schlichter konformer Abbildung mehrfachzusammenhängender Bereiche, Rec. Math. (Mat. Sbornik) N.S. vol. 2 (1937) pp. 37-64.

(c) Einige Koeffizientenabschätzungen fïr schlichte Funktionen, Rec. Math. (Mat. Sbornik) N.S. vol. 3 (1938) pp. 321-330.

(d) Interior problems of the theory of schlicht functions, Uspekhi Matematicheskikh Nauk vol. 6 (1939) pp. 26-89.

\footnotetext{
${ }^{7}$ An unpublished result of A. C. Schaeffer and the author.
} 
(e) Über Koeffizienten der schlichten Funktionen, Rec. Math. (Mat. Sbornik) N.S. vol. 12 (1943) pp. 40-47.

(f) On the theory of schlicht functions, Rec. Math. (Mat. Sbornik) N.S. vol. 18 (1946) pp. 167-179.

(g) On distortion theorems in the theory of conformal mapping, Rec. Math. (Mat. Sbornik) N.S. vol. 18 (1946) pp. 380-390.

4. H. Grötzsch, Über zwei Verschiebungsprobleme der konformen Abbildung, Preuss. Akad. Wiss. Sitzungsber. (1933) pp. 87-100.

5. H. Grunsky, Neue Abschätzungen zur konformen Abbildung ein- und mehrfachzusammenhängender Bereiche, Schrifter des mathematischen Seminars und des Instituts für angewandte Mathematik der Universität Berlin vol. 1 (1932).

6. J. E. Littlewood, (a) On inequalities in the theory of functions, Proc. London Math. Soc. (2) vol. 23 (1925) pp. 481-519.

(b) Theory of functions, Oxford University Press, 1944.

7. K. Löwner, Untersuchungen ïber schlichte konforme Abbildungen des Einheitskreises, Math. Ann. vol. 89 (1923) pp. 103-121.

8. E. Peschl, Zur Theorie der schlichten Funktionen, J. Reine Angew. Math. vol. 176 (1937) pp. 61-94.

9. R. M. Robinson, Bounded univalent functions, Trans. Amer. Math. Soc. vol. 52 (1942) pp. 426-449.

10. W. Rogosinski, Über positive harmonische Entwicklungen und typisch-reele Potenzreihen, Math. Zeit. vol. 35 (1932) pp. 93-121.

11. A. C. Schaeffer and D. C. Spencer, (a) The coefficients of schlicht functions, Duke Math. J. vol. 10 (1943) pp. 611-635. 125.

(b) The coefficients of schlicht functions II, Duke Math. J. vol. 12 (1945) pp. 107-

(c) The coefficients of schlicht functions III, Proc. Nat. Acad. Sci. U.S.A. vol. 32 (1946) pp. 111-116.

12. M. Schiffer, (a) Sur un problème d'extrémum de la représentation conforme, Bull. Soc. Math. France vol. 66 (1938) pp. 48-55.

(b) A method of variation within the family of simple functions, Proc. London Math. Soc. (2) vol. 44 (1938) pp. 432-449.

(c) Variation of the Green function and theory of the p-valued functions, Amer. J. Math. vol. 65 (1943) pp. 341-360.

(d) Hadamard's formula and variation of domain-functions, Amer. J. Math. vol. 68 (1946) pp. 417-448.

13. O. Toeplitz, Über die Fourier'sche Entwickelung positiver Funktionen, Rend. Circ. Mat. Palermo vol. 32 (1911) pp. 191-192.

StaNFord University 